\title{
Off-axis digital holographic microscopy: practical design parameters for operating at diffraction limit
}

\author{
Emilio Sánchez-Ortiga, ${ }^{1,3}$ Ana Doblas, ${ }^{1}$ Genaro Saavedra, ${ }^{1}$ \\ Manuel Martínez-Corral, ${ }^{1}$ and Jorge Garcia-Sucerquia ${ }^{2,4}$ \\ 'University of Valencia, 3D Imaging and Display Laboratory, Department of Optics, E-46100 Burjassot, Spain \\ ${ }^{2}$ Universidad Nacional de Colombia Sede Medellin, School of Physics, A.A: 3840-Medellin-050034, Colombia \\ ${ }^{3}$ e-mail: emilio.sanchez@uv.es \\ ${ }^{4}$ e-mail: jigarcia@unal.edu.co
}

Received 12 November 2013; revised 21 January 2014; accepted 12 February 2014; posted 24 February 2014 (Doc. ID 201109); published 26 March 2014

\begin{abstract}
The utilization of microscope objectives (MOs) in digital holographic microscopy (DHM) has associated effects that are not present in conventional optical microscopy. The remaining phase curvature, which can ruin the quantitative phase imaging, is the most evident and analyzed. As phase imaging is considered, this interest has made possible the development of different methods of overcoming its undesired consequences. Additionally to the effects in phase imaging, there exist a set of less obvious conditions that have to be accounted for as MOs are utilized in DHM to achieve diffraction-limit operation. These conditions have to be considered even in the case in which only amplitude or intensity imaging is of interest. In this paper, a thorough analysis of the physical parameters that control the appropriate utilization of MOs in DHM is presented. A regular DHM system is theoretically modeled on the basis of the imaging theory. The Fourier spectrum of the recorded hologram is analyzed to evaluate the performance of the DHM. A set of the criteria that consider the microscope features and the recording parameters to achieve DHM operation at the diffraction limit is derived. Numerical modeling and experimental results are shown to validate our findings. (C) 2014 Optical Society of America

OCIS codes: (090.1995) Digital holography; (110.0180) Microscopy; (110.2990) Image formation theory; (260.1960) Diffraction theory.

http://dx.doi.org/10.1364/AO.53.002058
\end{abstract}

\section{Introduction}

Digital holographic microscopy (DHM) has become a technique utilized widely for sample inspection, having many applications in different fields of science and technology. The capability for recovering the complex amplitude distribution scattered by the sample permits a postacquisition numerical refocus and a quantitative measurement of the sample phase. These are two of the features that make

$1559-128 \mathrm{X} / 14 / 102058-09 \$ 15.00 / 0$

(C) 2014 Optical Society of America
DHM a very versatile microscopy technique. With the benefit of such special characteristics, DHM is used for particle tracking [1], microelectromechanical systems (MEMS) characterization [2], and biological-samples inspection [3-5], among many other applications. The standard DHM system is based on a Mach-Zehnder interferometer that can be configured for operating in transmission or reflection modes, working in either the in-line or off-axis architecture.

When considering off-axis DHM, the approach to recover the complex amplitude distribution scattered by the specimen should include the following steps: 
a method to eliminate the twin image and the zero diffraction order, a numerical reference calculation, a refocusing algorithm, and, typically, a numerical phase masking in order to compensate the spherical phase distortion induced by the microscope objective (MO) [6]. The problem of the spherical phase introduced $\bar{b}$ y the MO has been treated in terms of the phase retrieval in quantitative phase-contrast imaging (QPI). This remaining phase has unwanted effects not only in the retrieved phase of the sample but also in the conditions of recording of the digital hologram to achieve diffraction-limited DHM. Many works have been performed in recent years in order to compensate this phase factor, both numerically and physically $[\underline{3}, \underline{6}-14]$. Computational methods are widespread and commonly used for compensating the all the defects introduced by the optical components in DHM systems [6,8-10,12]. For instance, in the case of the spherical distortion introduced by the imaging system, different numerical approaches eliminate that nuisance in the last step of the reconstruction process. In spite of the correction produced in the phase images, those methods cannot avoid the fact that such phase curvature is physically present in the imaging system during the recording process. As we show in this paper, that presence in the recording stage produces ruining effects that cannot be removed by computational methods. Some physical architectures permit us to minimize or even eliminate the phase curvature introduced by the imaging system in DHM. They reduce the number of calculations required in the reconstruction process, and they avoid the presence of quadratic phase factor in the recording stage $[11,13,14]$. An imaging system formed by an infinity-corrected MO in an afocaltelecentric configuration with a tube lens (TL) produces directly a plane wavefront in the object arm $[11,13,14]$. Another method is based on the utilization of the same spherical phase in the reference wave [3], by introducing an identical MO in the corresponding arm of the microscope. Additionally, other physical configurations, such as the use of liquid crystals, deformable mirrors, or a composite of lenses producing a plane wave in the object arm, can be used for avoiding the effect of the spherical phase factor.

The ruining effects that are present in the recording stage and cannot be removed by means of computational tools are analyzed in this work. A set of necessary physical conditions for the recording process in order to obtain the best quality possible in the reconstruction process is also given. Furthermore, we get the conditions over the microscope parameters [numerical aperture (NA) and magnifying factor] that must be accomplished in order to maintain a diffraction-limited image throughout the reconstruction stage.

Many works have studied the performance of offaxis digital holography [15-19]. As our DHM microscope operates in off-axis architecture, the conditions established in those works are assumed in our design. Some of the extra features added by the use of the MO in off-axis DHM have also been analyzed $[4,20]$. The former work analyzes the conditions that must be fulfilled to have high-resolution DHM as the holograms are reconstructed via the discrete Fresnel transform. The authors establish the required distances of recording for optimization of the lateral resolution. In the latter, the authors studied the spatial resolution and depth of field of the DHM as a function of the recording distance and also established a set of experimental conditions that help to optimize the performance of the DHM.

To contribute to the use of off-axis DHM, in this work we analyze the physical parameters that determine the diffraction-limited performance of this microscopy method. Our analysis considers the effects of the phase curvature of the imaging system on the amplitude contrast imaging. We focus on the derivation of a set of conditions that could be used as a map of the route to designing an off-axis DHM operating at the diffraction limit.

\section{Complete Model for Off-Axis DHM}

In this section we present a complete model for off-axis DHM. The model includes the imaging process and its effects in the hologram recording reconstruction. We analyze the consequences of the phase curvature induced by the MO, first in the imaging process and then in the hologram recording reconstruction. To this aim, we consider a regular imaging microscope formed by an infinity-corrected $\mathrm{MO}$ and a TL; see Fig. 1 . We will see that varying the distance between the MO and the TL can change the curvature of the remaining wavefront. The case of a finite-corrected MO can be deduced as a particular case of our general formalism.

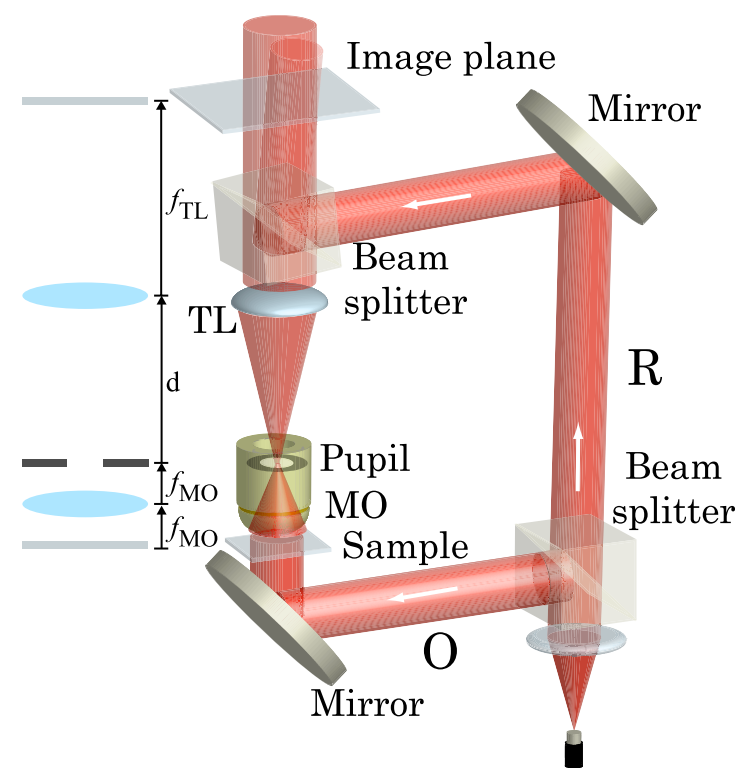

Fig. 1. Scheme of the off-axis DHM setup in transmission mode used in this work. 
A. Imaging Objects with Complex Amplitude Transmittance

The first step needed for the correct hologram recording is to obtain an enlarged, high-resolution, image of the microscopic specimen. In order to perform this task a conventional optical microscope is necessary, which is composed by the arrangement of a MO and a TL separated by a distance $d$, as illustrated in Fig. 1 . In the following, we assume that the aperture stop $p(\mathbf{x})$ of the MO is placed at its back focal plane (BFP). Let us place a given plane object with complex amplitude transmittance $O(\mathbf{x})$ in the front focal plane (FFP) of the MO. The amplitude distribution of the optical wavefield at the pupil plane is given by [21]

$$
U_{p}(\mathbf{x})=\exp \left(i 2 k f_{\mathrm{MO}}\right) \frac{-i}{\lambda f_{\mathrm{MO}}} \tilde{O}\left(\frac{\mathbf{x}}{\lambda f_{\mathrm{MO}}}\right) p(\mathbf{x})
$$

$\lambda$ being the wavelength of the illumination light, $f_{\text {MO }}$ the focal length of the MO in the image space, $\tilde{O}()$ the Fourier transform of the object amplitude transmittance, and $\mathbf{x}=(x, y)$ the transverse coordinates. For MOs with low NA, the propagation from the aperture plane can be accurately evaluated by use of the paraxial Fresnel diffraction integral. Using this formalism, it is possible to obtain a wavefield in the BFP of the TL; that is,

$$
\begin{aligned}
U^{\prime}(\mathbf{x})= & \frac{-1}{\lambda^{2} f_{\mathrm{MO}} f_{\mathrm{TL}}} \exp \left(i k L_{0}\right) \exp \left(i k \frac{1-d / f_{\mathrm{TL}}}{2 f_{\mathrm{TL}}}\left(|\mathbf{x}|^{2}\right)\right) \\
& \times \iint_{\mathfrak{R}^{2}} \mathrm{~d} \mathbf{x} \tilde{O}\left(\frac{\mathbf{x}_{0}}{\lambda f_{\mathrm{MO}}}\right) p\left(\mathbf{x}_{0}\right) \exp \left(-i \frac{2 \pi}{\lambda f_{\mathrm{TL}}}\left(\mathbf{x}_{0} \mathbf{x}\right)\right),
\end{aligned}
$$

where $L_{0}=2 f_{\mathrm{MO}}+d+f_{\mathrm{TL}}$.

Note that in the normal operation of an optical microscope equipped with an infinity-corrected $\mathrm{MO}$, the object is placed at the FFP of the $\mathrm{MO}$, and therefore the image appears at the BFP of the TL. For that reason, we name the later plane the image plane; see Fig. 1.

By using now the convolution theorem, the complex amplitude distribution outgoing from the system can be written as

$$
\begin{aligned}
U^{\prime}(\mathbf{x})= & \frac{1}{M} \exp \left(i k L_{0}\right) \exp \left(\frac{i k}{2 C}|\mathbf{x}|^{2}\right) \\
& \times\left\{O\left(\frac{\mathbf{x}}{M}\right) \otimes \tilde{p}\left(\frac{\mathbf{x}}{\lambda f_{\mathrm{TL}}}\right)\right\},
\end{aligned}
$$

$C=f_{\mathrm{TL}}^{2} /\left(f_{\mathrm{TL}}-d\right)$ being the radius of curvature of the spherical wavefront, and $M=-f_{\mathrm{TL}} / f_{\mathrm{MO}}$ the lateral magnification of the optical microscope.

It is worth noting that in conventional light microscopy, that is, when just the irradiance is captured, the multiplying spherical phase factor has no effect on the imaging process. However, that phase factor has a strong incidence in the performance of DHM, as will be analyzed in the following sections.

\section{B. Hologram Recording}

For recording a digital hologram in the off-axis architecture, the digital camera is placed at a distance $z$ from the image plane. Then, the interference between the propagated field of the object wave $U^{\prime}(\mathbf{x})$ and a tilted plane reference wavefield is recorded. The digital hologram in the recording plane is given by

$$
I_{z}(\mathbf{x})=\left|U_{z}(\mathbf{x})\right|^{2}+|R(\mathbf{x})|^{2}+U_{z}(\mathbf{x}) R^{*}(\mathbf{x})+U_{z}^{*}(\mathbf{x}) R(\mathbf{x}),
$$

where $*$ represents the complex conjugate. $R(\mathbf{x})=$ $\exp [i(\mathbf{k} \cdot \mathbf{x})]$ is the reference plane wave, in which $\mathbf{k}=\left(k_{x}, k_{y}\right)$ is the wavevector, and

$$
U_{z}(\mathbf{x})=\frac{i}{\lambda z} \exp (i k z) U^{\prime}(\mathbf{x}) \otimes \exp \left[\frac{i k}{2 z}|\mathbf{x}|^{2}\right] .
$$

is the free-space propagated object wave.

The digital hologram, Eq. (4), is composed of four terms that encode the information of interest. For simplicity, we do not consider explicitly the discretization effects, but they will be accounted for in following sections. In off-axis DHM the digital hologram must be recorded such that the four terms can be isolated. This process, called Fourier filtering [21], is only possible if the wavevector $\mathbf{k}=\left(k_{x}, k_{y}\right)$ produces enough lateral shifting. This shifting must be evaluated in the spectral domain. To this end we calculate the Fourier transform of the digital hologram, which is given by

$\tilde{I}_{z}(\mathbf{u})=\mathrm{DC}(\mathbf{u})+\tilde{U}_{z}(\mathbf{u}) \otimes \delta(\mathbf{u}+\mathbf{k})+\tilde{U}_{z}^{*}(\mathbf{u}) \otimes \delta(\mathbf{u}-\mathbf{k})$,

where

$$
\mathrm{DC}(\mathbf{u})=\mathfrak{s}\left\{\left|U_{z}(\mathbf{x})\right|^{2}+|R(\mathbf{x})|^{2}\right\}
$$

is the zero-order diffraction term (usually named the DC term), with $\mathfrak{I}$ denoting the Fourier transform. This term is placed, of course, at the center of the spectrum.

The other two terms $\tilde{U}_{z}(\mathbf{u}) \otimes \delta(\mathbf{u}+\mathbf{k})$ and $\tilde{U}_{z}^{*}(\mathbf{u}) \otimes$ $\delta(\mathbf{u}-\mathbf{k})$ exhibit a displacement proportional to the wavevector $\mathbf{k}$; these terms are named the +1 and -1 diffraction orders, respectively [22].

The direction of the wavevector also controls the spatial frequency of the digital hologram. In the forthcoming analysis we consider that the maximum spatial frequency of the digital hologram fulfills the Nyquist requirements [22]. This condition dictates the maximum angle, $\phi_{\max }$, that can exist between the known reference wave and the object wave $[\underline{15}, \underline{16}, \underline{18}]$, namely,

$$
\phi_{\max }=\frac{\lambda}{2 \Delta p},
$$

with $\Delta p$ the pixel size of the digital camera. 
Initially, we assume that the shifting introduced by $\mathbf{k}$ is enough to isolate the +1 term from the others. In such a case we can focus our study on that term. This term can be written as

$$
\tilde{U}_{z}(\mathbf{u})=\tilde{U}^{\prime}(\mathbf{u}) \exp \left[-i \pi \lambda z|\mathbf{u}|^{2}\right]
$$

where some irrelevant constants have been omitted. As can be seen, the propagation distance $z$ affects only the phase of the term. The Fourier transform in the image plane of the object wave is given by

$$
\tilde{U}^{\prime}(\mathbf{u})=\left[\exp \left(-i \pi \lambda C|\mathbf{u}|^{2}\right) \otimes\left\{\tilde{O}(M \mathbf{u}) p\left(\lambda f_{\mathrm{TL}} \mathbf{u}\right)\right\}\right]
$$

As we will see in the next section, the curvature of the remaining spherical wavefront, $C^{-1}$, affects not only the shape but also the size of the diffraction order. This will influence the capacity of isolating the orders, and therefore the final resolution limit achievable after the rendering process.

\section{Effects of the Phase Curvature on the Spatial Filtering of the Hologram}

In off-axis DHM the sample information is encoded in the +1 and -1 diffraction orders. This feature imposes the need for separating them from the DC diffraction order. That separation is done with the proper angle of the $\mathbf{k}$ vector, namely the angle between the reference and the object waves. The spreading of the \pm 1 diffraction orders limits the utilization of the space bandwidth of the hologram. For this reason it is necessary to study how the design of the optical arrangement influences the shape and the size of those terms.

\section{Limit Case $C \rightarrow \infty$ : Telecentric Mode}

Let us consider the case in which the radius of curvature, $C$, of the remaining wavefront approaches to infinity, that is, when a plane wave comes out from the microscope. In such a case,

$$
\mathrm{DC}(\mathbf{u})=\delta(\mathbf{u})+\left\{\tilde{O}(M \mathbf{u}) p\left(\lambda f_{2} \mathbf{u}\right)\right\} \otimes\left\{\tilde{O}(M \mathbf{u}) p\left(\lambda f_{2} \mathbf{u}\right)\right\}^{*},
$$

and

$$
\tilde{U}_{z}(\mathbf{u}, C \rightarrow \infty)=\left\{\tilde{O}(M \mathbf{u}) p\left(\lambda f_{2} \mathbf{u}\right)\right\} \exp \left[-i \pi \lambda z|\mathbf{u}|^{2}\right] .
$$

From Eq. (11) one can note that the size and shape of the DC term do not depend on the curvature $C$. Its size is twice the size of the pupil of the imaging system, since it is given by its autocorrelation $[17,18]$.

At this point we can establish the conditions that optimized the utilization of the MO by making the best use of the space bandwidth. To this aim we consider a circular pupil function of $r$ radius measured in the pupil plane. Mathematically, the pupil function appearing in the Fourier space is

$$
p\left(\lambda f_{\mathrm{TL}} \mathbf{u}\right)=\operatorname{circ}\left(\frac{\lambda f_{\mathrm{TL}}}{r}|\mathbf{u}|\right) .
$$

The radius of the pupil function in the Fourier space is $\rho=r /\left(\lambda f_{\mathrm{TL}}\right)$. The radius of the pupil written as a function of NA and lateral magnification of the $\mathrm{MO}$ is $\rho=\mathrm{NA} /(\lambda M)$ [23]. From the consideration of this size, we can select the appropriate MO that can be utilized in a particular DHM setup, such that the use of space bandwidth is optimized.

Let us consider the space-bandwidth product of a square camera with $N \times N$ pixels of $(\Delta p, \Delta p)$ of size. The solid lines in Fig. 2 represent the borders of the terms of the digital-hologram spectrum for the limit case in which the maximum space bandwidth is utilized to record the whole information of the wavefield. This condition is accomplished when the imaging system works in the telecentric mode (TM), provided that the diagonal

$$
D=2 \rho_{\mathrm{TM}}(3+\sqrt{2}) .
$$

The sampling pitch in the Fourier domain is given by the relationship $\Delta u=1 /(N \Delta p)$; then the diagonal size is $D=\sqrt{2} / \Delta p$. Taking into account these figures, the radius of the pupil that optimized the use of the space bandwidth for the TM is

$$
\rho_{\mathrm{TM}} \leq \frac{1}{\sqrt{2}(\sqrt{2}+3) \Delta p},
$$

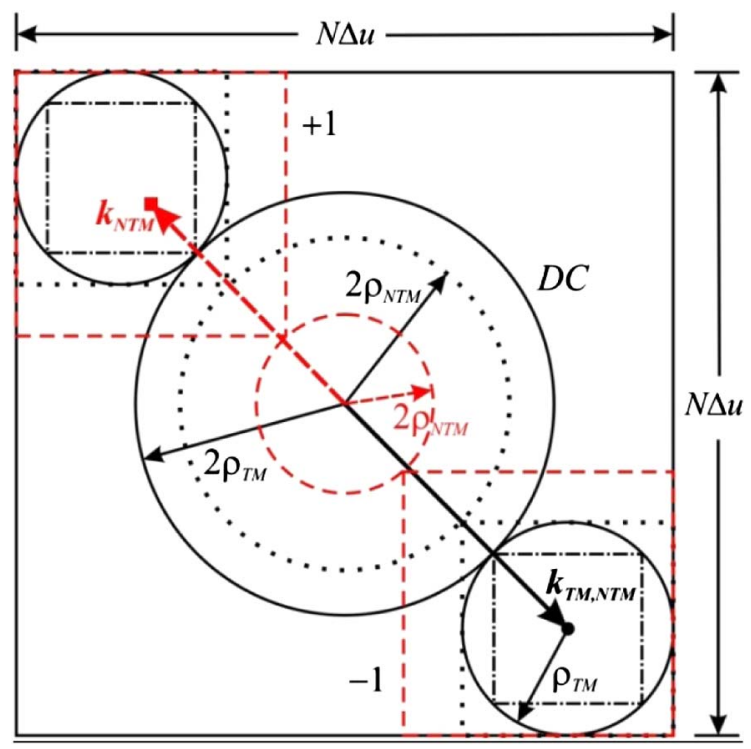

Fig. 2. Fourier transform of the digital hologram. The sizes and shapes of diffraction orders are illustrated. The DC diffraction order is placed at the center of the Fourier spectrum of the digital hologram; its size indicates the achievable resolution of the DHM. The shape and size of the +1 and -1 diffraction orders are controlled by the value of the wavefront curvature $C$. The solid lines correspond to the TM operation. The dotted/dashed lines are both for the nontelecentric mode (NTM) with different values of $C$. 
which leads to

$$
\frac{\mathrm{NA}}{M} \leq \frac{\lambda}{\sqrt{2}(\sqrt{2}+3) \Delta p} .
$$

Equation (16) dictates limit values of the NA and the magnification of the MO that can be used in an optimized DHM operating in TM. Owing to this condition being independent of the number of pixels, one can conclude that the number of pixels determines only the field of view (FOV) of the microscope. However there exist a minimum number of pixels that need to be taken, such that the Fourier transform of the diffraction order can be recovered without distortion. We have experimentally found that number of the order of 128 pixels. From this rule of thumb one can conclude that the minimum FOV in the object plane equals $128 \Delta p / M$.

The condition expressed by Eq. (16) assumes the complete separation of the +1 and $\overline{-1}$ diffraction orders from the DC. As shown in Fig. 2, this separation is achieved on the condition that $\left|\mathbf{k}_{\mathrm{TM}, \mathrm{NTM}}\right| \geq 3 \rho_{\mathrm{TM}}$. For a square digital sensor the optimized use of the space bandwidth is obtained for $k_{x}=k_{y}$. For this case we obtain

$$
\frac{\mathrm{NA}}{M} \leq \frac{2 \sqrt{2} \pi}{3} \sin \phi,
$$

where $\phi$ is the angle of inclination of the wavevector with respect to the $x$ and $y$ axes, namely the angle between the reference and object wave along the $x$ and $y$ axes with respect to the direction of propagation. Equations (8) and (17) can be combined to conclude that the angle between the reference and object waves must be

$$
\sin ^{-1}\left(\frac{3}{2 \sqrt{2} \pi} \frac{\mathrm{NA}}{M}\right) \leq \phi \leq \frac{\lambda}{2 \Delta p} .
$$

Whenever the condition states on Eqs. (16) and (18) are met, the DHM operating in TM $[11,1 \overline{3,14}]$ can be considered as diffraction limited because there is enough space bandwidth in the Fourier domain for filtering the +1 and -1 diffraction orders without losing spatial frequencies or introducing noise proceeding from the DC diffraction order.

It should be pointed out that the usable space bandwidth of the hologram can be further enhanced by reducing the effect of the DC order by means of the utilization of nonlinear filters [24]. This nonlinear approach can extend the usable space bandwidth up to $N \Delta u / 2$ along each direction. The trade-off for this improvement is that the object amplitude must be weak in comparison with the reference wave; namely the method can be applied only for imaging weak scatters. As the method we present in this work resorts in a simple binary filter that matches the pupil size in the reciprocal space, it has no restriction in terms of the objects to be imaged.

\section{Phase Curvature C Taking Any Value: Nontelecentric Mode}

Let us study the case in which the imaging system operates in nontelecentric mode (NTM), so that $C$ can take any value. As can be seen from Eq. (11), neither the shape nor the size of the DC term is affected by the phase curvature of the wavefront. However, both the +1 and -1 diffraction orders are modified by a convolution term that is dependent on $C$. As can be read from Eq. (10), both orders can be understood as diffraction patterns of the Fourier transform of the image of the object. This behavior is somewhat undesired inasmuch as it makes the spatial filtering tougher and dependent on parameters such as the pixel size, number of pixels, value of curvature of the wavefront, and reconstruction distance.

To illustrate that difference, in Fig. 3 we show the Fourier transform of modeled digital holograms recorded for two different values of $C$. While Fig. 3(a) shows the spectrum of a digital hologram of a USAF test target recorded in the TM, Fig. 3(b) shows the corresponding spectrum for $C=2 \overline{66} \mathrm{~mm}$, NTM. For the TM the \pm 1 terms are fitted inside circles with radii equal to the telecentric pupil $\rho_{\mathrm{TM}}$. However, in the NTM those terms are bounded by the squares; see Fig. $3(\mathrm{~b})$. All the simulations and computations were done using MATLAB.

That difference in the behavior of the diffraction orders of the digital-hologram spectrum makes it necessary to study how their size changes with the value of $C$. One can consider that the maximum spread of +1 and -1 diffraction orders occurs within a window with a size given by the propagation of the central pixel of the spectrum, which is the most diffractive element in the spectrum. The elementary central pixel in the Fourier domain can be written as $\operatorname{rect}(u / \Delta u)$. Then, introducing this term into Eq. (9) and expanding the convolution operation, we obtain

$$
\begin{aligned}
\tilde{U}_{z}(\mathbf{u})= & \exp \left(-i \pi \lambda(C+z)|\mathbf{u}|^{2}\right) \\
& \times \mathfrak{I}\left(\operatorname{rect}\left(\mathbf{u}_{0} / \Delta u\right) \exp \left(-i \pi \lambda C\left|\mathbf{u}_{0}\right|^{2}\right)\right)_{u_{0}=\lambda C u} .
\end{aligned}
$$

The spreading of the diffraction order can be computed by evaluating the change of scale implicit in
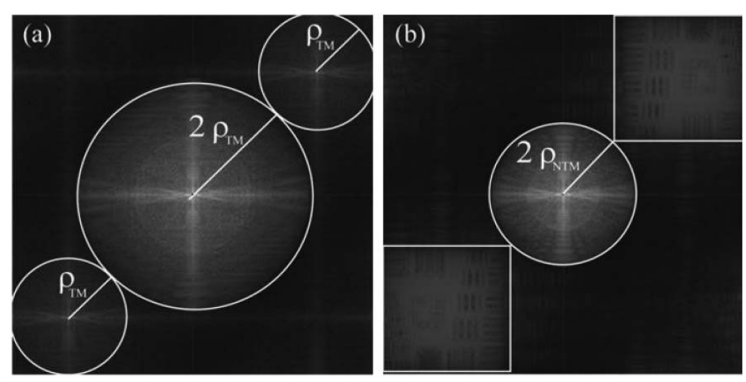

Fig. 3. Spectrum of modeled digital holograms of a USAF test target for (a) telecentric mode, $C \rightarrow \infty$, and (b) NTM, spherically phase distorted case for $C=266 \mathrm{~mm} \quad(d=50 \mathrm{~mm}$ and $f_{\mathrm{TL}}=200 \mathrm{~mm}$ ). See text for further details. 
Eq. (19). This calculation is somehow equivalent to the computation of the size of the zero order of diffraction in numerical Fresnel propagation [25]. Taking into account the discretization and argument of the exponential factor in front of the Fourier transform, we obtain

$$
\Delta u=\frac{N^{2} \Delta p^{2}(C+z)}{\lambda C^{2}} \Delta u_{0}
$$

For simplicity, in following analysis we neglect $z$ with respect to $C$, without forgetting that the larger $z$, the lower the effect of $C$. Under the above approximation one considers that the digital hologram is recorded in a plane close enough to the image plane so that we can use the angular spectrum [26] for its numerical refocusing. By taking this approximation into account the magnifying factor Eq. (20) simplifies as

$$
\Delta u=\frac{N^{2} \Delta p^{2}}{\lambda C} \Delta u_{0}
$$

We have verified the spreading of the +1 and -1 diffraction orders by means of computer modeling and experimental results. We simulate the spreading according with Eq. (19) and the size predicted by the magnifying factor Eq. (21), for different values of $C$. In Fig. 4(a), while the dashed white lines represent the magnifying factor, the gray cone-shaped intensity in between them is the modulus of Eq. (19); this simulation was performed for the experimental parameters we have utilized, $N=1024, \Delta p=6.9 \mu \mathrm{m}$, and $\lambda=632.8 \mathrm{~nm}$. For the experimental validation we have recorded a set of digital holograms with no object inserted for different values of $C$. The size of the +1 diffraction order is plotted Fig. 4 (b) as the curvature varies. The values of those sizes have been fitted in $(N \Delta p)^{2} /(2 \lambda C)$ to plot the white dotted line in Fig. $4(\mathrm{~b})$. The theoretical value for the slope, taking into account that we are measuring half of the window, is $3.9496 \times 10^{4} \mathrm{~mm}$. The measured slope for the magnifying function is $3.94 \times 10^{4} \mathrm{~mm}$, which matches the theoretical value. In the fitting the correlation coefficient was 0.996 . From this result we can state that the expansion of the +1 order due to

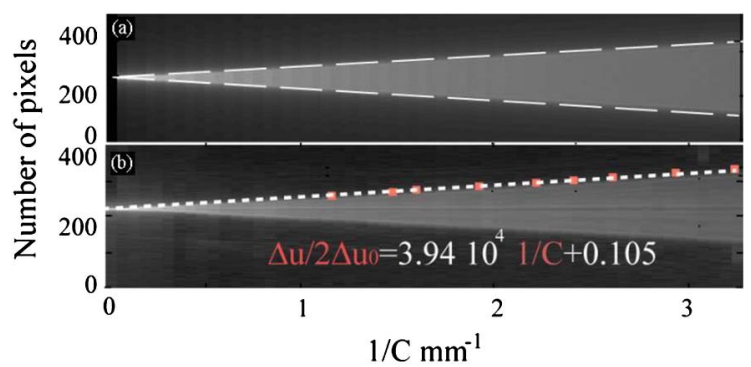

Fig. 4. Spreading of the +1 diffraction orders as the value of $C$ changes. (a) shows the results from computer modeling. The experimental results are shown in (b). The behavior is identical for the -1 diffraction order. the presence of curvature in the object arm is ruled by Eq. (21).

Owing to the size of the nonpropagated central pixel is $\Delta u_{0}=1 / N \Delta p$; from Eq. (21) the size of the +1 and -1 diffraction orders varies as $N \Delta p /(\lambda C)$ along each side of the squares that bound them. The square-shaped diffraction orders, with sides of the above size, have to be fitted in the space bandwidth with the circular DC term with no overlapping, as illustrated in Fig. $\underline{2}$. In that figure the squares made of nonsolid lines correspond to the +1 and -1 diffraction orders for the different values of $C$ we considered. Three different values for $C$, namely three squares with different sides, are analyzed. For determining the boundaries here presented, the reader should considerer the size of the diffracted order and match it with the corresponding $\rho_{\mathrm{TM}}$ illustrated in Fig. 2:

(i) $C \geq\left(\sqrt{2} N \Delta p / 2 \lambda \rho_{\mathrm{TM}}\right)$. This value of $C$ allows for fitting the complete square diffraction order inside a circle with radius $\rho_{\mathrm{TM}}$; see the squares made of dashed dotted lines in Fig. 2. This value of $C$ allows for maintaining the diffraction-limited performance of the DHM at the same level as the TM because $\rho_{\mathrm{NTM}}=\rho_{\mathrm{TM}}$.

(ii) $\left(\sqrt{2} N \Delta p / 2 \lambda \rho_{\mathrm{TM}}\right)>C \geq\left(N \Delta p / 2 \lambda \rho_{\mathrm{TM}}\right)$. As the value of $C$ diminishes, the DC diffraction order has to shrink for allowing the fitting of the square diffraction orders; see the dotted circle and dotted squares in Fig. 2. In this case the achievement of the diffraction-limited performance of the DHM imposes a diminishing of the resolution of the microscope in comparison with case (i); the new value for the pupil of the microscope is $\rho_{\mathrm{NTM}} \approx(\sqrt{2} / 4 \Delta p)-$ $(\sqrt{2} N \Delta p-2 \lambda C)$, which after some direct algebra can be shown to be smaller than that for the TM.

In the two former cases for the values of $C$, the magnitude of the wavevector $\mathbf{k}$ remains equal to its value in the TM. This means that the angle between the reference and object waves still fulfills Eq. (18). However, as illustrated in Fig. 2, the wavefront curvature can take even smaller values but under the condition that the magnitude of the wavevector $\mathbf{k}$ diminishes to allow the fitting of the diffraction orders in the space bandwidth. The magnitude of the new wavevector $\mathbf{k}$ must be

$$
\left|\mathbf{k}_{\mathrm{NTM}}\right| \leq \frac{\sqrt{2}}{2}\left(\frac{1}{\Delta p}-\frac{N \Delta p}{\lambda C}\right) .
$$

While Eq. (22) imposes the limit for the minimum angle between the reference and object waves that guarantees no overlapping of the diffraction orders, Eq. (8) determines the maximum angle. If the value of $\left|\mathbf{k}_{\mathrm{NTM}}\right|$ changes according with Eq. (22), the nontelecentric DHM can operate at the range presented in (iii).

(iii) $C<\left(N \Delta p / 2 \lambda \rho_{\mathrm{TM}}\right)$. As the $C$ value is reduced further from the value established in (ii), the DC diffraction order has to be shrunk to allow the fitting of the 
enlarged +1 and -1 diffraction orders. As mentioned above the angle between the reference and object waves has to be smaller in comparison with that for the TM. The new allowable value for the pupil of the DHM is $\rho_{\mathrm{NTM}} \leq(\sqrt{2} / 4 \Delta p)-(\sqrt{2} N \Delta p / 2 \lambda C)$, which is quite smaller than $\rho_{\mathrm{TM}}$.

In summary, the diffraction-limit performance of the DHM can be achieved whatever wavefront curvature is chosen for the object wave, provided that the proper spatial filtering of the DC, +1 , and -1 diffraction orders is possible. However, the reader must be aware that the higher the value of $C$, the better the resolution of the microscope. To make possible the proper spatial filtering, no overlapping of the diffraction orders has to be guaranteed. In the case in which the orders are overlapped, the +1 and -1 diffraction orders cannot be isolated completely from the DC diffraction order, which introduces noises and contrast variations in the reconstructed holograms.

According with (i), (ii), and (iii), once one has chosen a digital camera and wavelength, there is a tight dependence of the radius of the usable pupil of the microscope with the curvature the object wavefront $C$, namely with the achievable resolution of the DHM. For a better illustration of this dependence, in Fig. $\underline{5}$ we have plotted the ratio $\rho_{\mathrm{NTM}} / \rho_{\mathrm{TM}}$ as a function of $1 / C$. We have utilized for this numerical model $\lambda=632.8 \mathrm{~nm}$, because this is the wavelength that we have utilized in our experiment. The set of pair values, pixel size, and number of pixels, shown in Fig. 5, has been chosen to consider the utilization of diverse digital cameras commercially available.

From Fig. $\underline{5}$, one can conclude that the resolution of the telecentric and nontelecentric DHM coincides for a threshold value of $1 / C$. We can as well conclude that as the extension of the digital recording increases, the value of $C$ for which the telecentric and nontelecentric DHM have the same resolution also increases. In particular, for the camera utilized in this work, $N=1024$ and $\Delta p=6.9 \mu \mathrm{m}$, the threshold up to which both modes operate at the same resolution is $1 / C=0.0022 \mathrm{~mm}^{-1}$. For larger values of $1 / C$ the nontelecentric DHM exhibits a lower resolution than the telecentric DHM, if one is interested in having no overlapping of the diffraction orders such

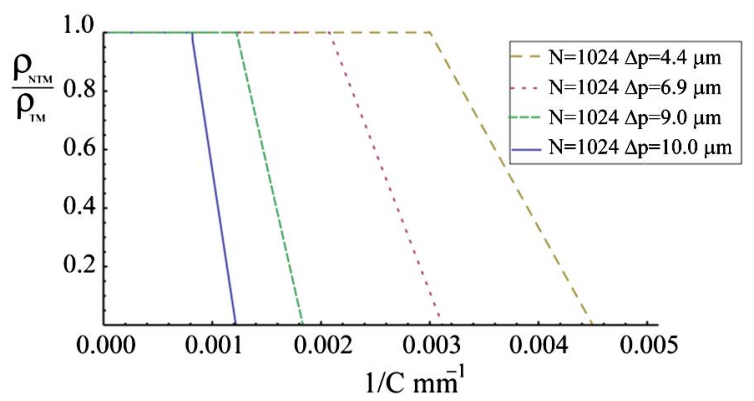

Fig. 5. Variation of $\rho_{\mathrm{NTM}} / \rho_{\mathrm{TM}}$ as a function of $1 / C$. The plot is illustrated for $\lambda=632.8 \mathrm{~nm}$ and different numbers of pixels and pixel sizes. that any perturbation of the reconstructed image is avoided. This condition is illustrated in the following section with experimental results.

\section{Design Example}

The results presented above for operating a DHM at the resolution limit have been utilized for validating their usefulness. The light from a He-Ne laser is utilized for building the setup illustrated in Fig. 1 . The chosen camera is a $1024 \times 1024$ pixel CCD technology from the company IDS-Imaging. Each square pixel has a $6.9 \mu \mathrm{m}$ side, which renders it to a maximum angle between the reference and object waves [Eq. (8)] of about $3^{\circ}$. From Eq. (16), for the TM the characteristics of the MO must meet the condition NA $/ M=0.0147$. To validate these figures, we started using a low-NA MO that permits the imaging of a negative 1951 USAF resolution target at the diffraction limit. We have chosen a 2.5x/0.075 MO for which NA $/ M=0.015$.

In the setup of Fig. 1 we have inserted a variable aperture to control the size of the effective pupil of the imaging system; the control of the pupil allows for achieving the conditions imposed in the above paragraphs for operating the DHM at the diffraction limit with no overlapping of the diffraction orders. The Fourier spectra of the recorded holograms along with the corresponding reconstructed images are shown in Fig. 6. In Fig. 6(a) the DHM is tuned to operate in TM. For this mode the size of the pupil in the real space is of the order of $5 \mathrm{~mm}$. In this configuration the object wavefront is essentially a plane wave, $C \rightarrow \alpha$. For this setup, as shown in Fig. 6(a), righthand side, the resolution of the system reaches up to element 2 of group $6(71.8 \mathrm{lp} / \mathrm{mm})$ in the test target. When the DHM operates in NTM, the size of the +1 and -1 diffraction orders rises and the DC diffraction order has to be shrunk. This shrinking means a reduction of the pupil size, which imposes a lower resolution than that for the TM. Figures 6(b) and 6(c) illustrate two situations in which $\mathrm{DHM}$ operates in NTM. The foreseen diminishing of the spatial resolution is illustrated clearly. In the images at the right-hand side of Figs. $\underline{6(\mathrm{~b})}$ and $\underline{6(\mathrm{c})}$, it is clear that smallest resolved elements are 5 group 5 $(50.8 \mathrm{lp} / \mathrm{mm})$ and 3 group $4(20.16 \mathrm{lp} / \mathrm{mm})$, in that order. For Fig. 6(b) the value of $C=333.3 \mathrm{~mm}$; in Fig. $6(\mathrm{c}) C=25 \overline{\mathrm{mm}}$.

After validating the design parameters presented above, a similar experiment to illustrate the conditions of operation of DHM with biological samples was also performed. In this case we imaged the object by using a $10 x / 0.45 \mathrm{MO}$. A section of a sunflower leaf was imaged with the DHM operating at the same curvatures of the object wavefront that were utilized in the experiment illustrated formerly. For the TM the reconstructed image shows details that are no longer visible or become fuzzy in the images for the nontelecentric operation; see Figs. 7(a), 7(b), and 7(c), in that order. The values of the curvature radio were $C=333.3 \mathrm{~mm}$ for Fig. $7(\mathrm{~b})$ and $C=250 \mathrm{~mm}$ for 

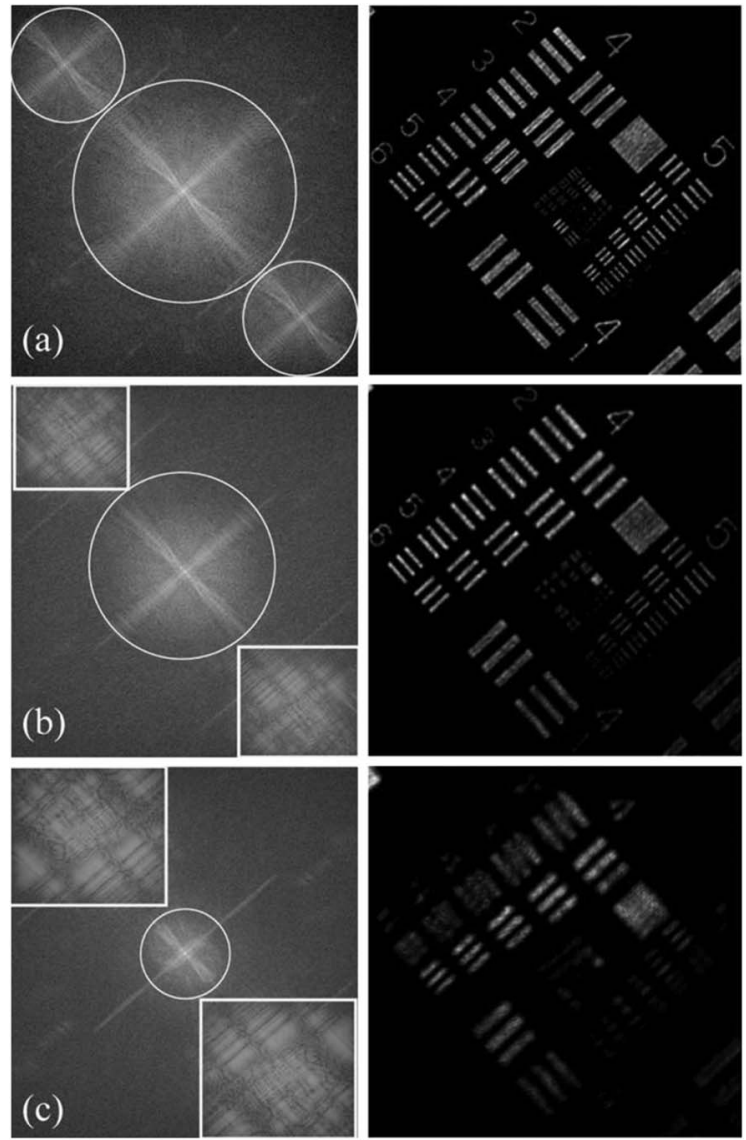

Fig. 6. DHM operating at diffraction limit for different modes of operation. Fourier spectrum of the recorded hologram at the lefthand side and a zoomed view of the corresponding reconstruction image at the right-hand side. (a) corresponds to TM. (b) and (c) are for nontelecentric operation of the DHM.

Fig. 7(c). It is also clear that as the value of $C$ decreases, the capability of the microscope to resolve smaller details decreases too; namely the resolution power of the microscope is reduced as the value of $C$ diminishes. The zoomed-in rectangular areas in Fig. 7 are shown for validating the above sentences. As can be seen, the shape of the sample strongly affects the sharpness of the Fourier transform. In that case the sample is mainly composed of low frequencies. In consequence, the spreading of the spectrum owing to the presence of the DC order is smoother than in the case of the USAF test. Then the effect due to the reduction of the pupil is not that critical as in the case of using samples composed mostly of high spatial frequencies. However, it is worthwhile it to apply the optimal parameters to the design for being sure that the system is providing the best quality images independently of the sample.

The perfect fitting with no overlapping of the +1 , DC, and -1 diffraction orders in the available space bandwidth allows for the appropriate spatial filtering and reconstruction of the digital holograms. If for the contrary there exists overlapping of the diffraction orders, the performance of the DHM will depend strongly on the characteristics of the sample

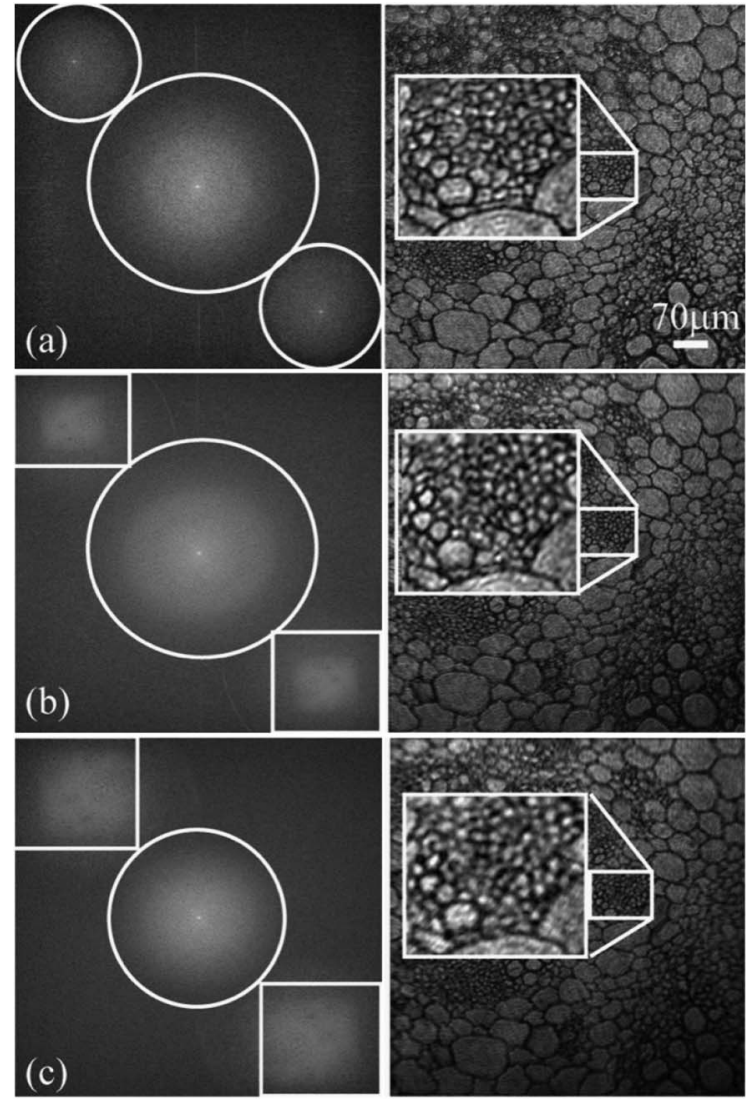

Fig. 7. DHM operating at diffraction limit applied to a biological sample. Fourier spectrum of the recorded hologram at the left-hand side and the corresponding reconstruction image at the right-hand side. (a) corresponds to TM. (b) and (c) are for nontelecentric operation of the DHM.

under study. For slightly diffractive samples, minimum perturbations of the +1 and -1 diffraction orders come from the DC order; hence minimum flaws are visible in the reconstructed images. In the opposite case, if the DC diffraction order introduces strong distortions on the +1 and -1 diffraction orders, the reconstructed images present important distortions, especially in their contrast. A detailed study of these effects is currently ongoing by our group.

\section{Conclusion}

An analysis of the effects that the use of MOs has in off-axis DHM has been presented. The analysis is supported on the study of the Fourier spectrum of the digital hologram recorded off-axis. For the recording of the hologram, a tunable imaging system has been utilized. The imaging system, composed of an infinity-corrected MO and a TL, can be tuned to have telecentric or nontelecentric recording of the hologram. The results showed that the nontelecentric DHM presents limitations in both quantitative phase imaging and amplitude contrast imaging. The former has been previously analyzed by ours and many other groups. Then our study here is focused on amplitude contrast imaging exclusively. 
The presence of the residual curvature phase in the nontelecentric imaging system imposes a set of conditions that must be accounted for to minimize the distortion of the reconstructed holograms. We have found that the \pm 1 terms in the Fourier spectrum of the hologram broaden as a function of the inverse of the curvature radius of the remaining phase factor. This broadening reduces the possibilities of proper spatial filtering of the DC term and twin image, which deteriorate the hologram reconstruction. From a thorough theoretical analysis we have derived a set of criteria that maximize the use of $\mathrm{MO}$ in off-axis DHM. These criteria have been tested to show the validity of our findings. From these results we can conclude that there is trade-off between the optical imaging system utilized for the recording of the hologram and the digital camera, which controls the performance of the complete DHM. Following these design criteria optimizes the use of $\mathrm{MO}$ in off-axis DHM.

This work was supported by the Ministerio de Ciencia e Innovación, Spain (Grant DPI201232993), and also by Generalitat Valenciana (Grant PROMETEO2009-077). A. Doblas acknowledges a predoctoral fellowship from the University of Valencia. J. Garcia-Sucerquia gratefully acknowledges the Visiting Scholar Fellowship from the Universidad de Valencia, Colciencias Grant No. 110205024, and UNAL Grant Nos. 110201003 and 110201004 .

\section{References}

1. F. C. Cheong, B. J. Krishnatreya, and D. G. Grier, "Strategies for three-dimensional particle tracking with holographic video microscopy," Opt. Express 18, 13563-13573 (2010).

2. P. Ferraro, S. De Nicola, G. Coppola, A. Finizio, D. Alfieri, and G. Pierattini, "Controlling image size as a function of distance and wavelength in Fresnel-transform reconstruction of digital holograms," Opt. Lett. 29, 854-856 (2004).

3. C. Mann, L. Yu, C.-M. Lo, and M. Kim, "High-resolution quantitative phase-contrast microscopy by digital holography," Opt. Express 13, 8693-8698 (2005).

4. D. Carl, B. Kemper, G. Wernicke, and G. von Bally, "Parameter-optimized digital holographic microscope for high-resolution living-cell analysis," Appl. Opt. 43, 65366544 (2004).

5. S. Seo, T. W. Su, A. Erlinger, and A. Ozcan, "Multi-color LUCAS: lensfree on-chip cytometry using tunable monochromatic illumination and digital noise reduction," Cell. Molec. Bioeng. 1, 146-156 (2008).

6. E. Cuche, P. Marquet, and C. Depeursinge, "Simultaneous amplitude-contrast and quantitative phase-contrast microscopy by numerical reconstruction of Fresnel off-axis holograms," Appl. Opt. 38, 6994-7001 (1999).

7. P. Ferraro, S. De Nicola, A. Finizio, G. Coppola, S. Grilli, C. Magro, and G. Pierattini, "Compensation of the inherent wave front curvature in digital holographic coherent microscopy for quantitative phase-contrast imaging," Appl. Opt. 42, 1938-1946 (2003).
8. T. Colomb, E. Cuche, F. Charrière, J. Kühn, N. Aspert, F. Montfort, P. Marquet, and C. Depeursinge, "Automatic procedure for aberration compensation in digital holographic microscopy and applications to specimen shape compensation," Appl. Opt. 45, 851-863 (2006).

9. T. Colomb, J. Kühn, F. Charrière, C. Depeursinge, P. Marquet, and N. Aspert, "Total aberrations compensation in digital holographic microscopy with a reference conjugated hologram," Opt. Express 14, 4300-4306 (2006).

10. T. Colomb, F. Montfort, J. Kühn, N. Aspert, E. Cuche, A. Marian, F. Charrière, S. Bourquin, P. Marquet, and C. Depeursinge, "Numerical parametric lens for shifting, magnification, and complete aberration compensation in digital holographic microscopy," J. Opt. Soc. Am. A 23, 3177-3190 (2006).

11. E. Sánchez-Ortiga, P. Ferraro, M. Martínez-Corral, G. Saavedra, and A. Doblas, "Digital holographic microscopy with pure-optical spherical phase compensation," J. Opt. Soc. Am. A 28, 1410-1417 (2011).

12. K. W. Seo, Y. S. Choi, E. S. Seo, and S. J. Lee, "Aberration compensation for objective phase curvature in phase holographic microscopy," Opt. Lett. 37, 4976-4978 (2012).

13. E. Sánchez-Ortiga, A. Doblas, M. Martínez-Corral, G. Saavedra, and J. Garcia-Sucerquia, "Aberration compensation for objective phase curvature in phase holographic microscopy: comment," Opt. Lett. 39, 417 (2014).

14. A. Doblas, E. Sánchez-Ortiga, M. Martínez-Corral, G. Saavedra, P. Andrés, and J. Garcia-Sucerquia, "Shift-variant digital holographic microscopy: inaccuracies in quantitative phase imaging," Opt. Lett. 38, 1352-1354 (2013).

15. T. M. Kreis, "Frequency analysis of digital holography," Opt. Eng. 41, 771-778 (2002).

16. L. Xu, X. Peng, Z. Guo, J. Miao, and A. Asundi, "Imaging analysis of digital holography," Opt. Express 13, 2444-2452 (2005).

17. P. Picart and J. Leval, "General theoretical formulation of image formation in digital Fresnel holography," J. Opt. Soc. Am. A 25, 1744-1761 (2008).

18. N. Verrier and M. Atlan, "Off-axis digital hologram reconstruction: some practical considerations," Appl. Opt. 50, H136-H146 (2011).

19. D. P. Kelly, J. J. Healy, B. M. Hennelly, and J. T. Sheridan, "Quantifying the 2.5D imaging performance of digital holographic systems," J. Eur. Opt. Soc. Rapid Pub. 6, 11034 (2011).

20. D. Claus and D. Iliescu, "Optical parameters and spacebandwidth product optimization in digital holographic microscopy," Appl. Opt. 52, A410-A422 (2013).

21. E. Cuche, P. Marquet, and C. Depeursinge, "Spatial filtering for zero-order and twin-image elimination in digital off-axis holography," Appl. Opt. 39, 4070-4075 (2000).

22. J. W. Goodman, Introduction to Fourier Optics (Roberts, 2005).

23. M. Martínez-Corral and G. Saavedra, "The resolution challenge in 3D optical microscopy," in Progress in Optics, E. Wolf, ed. (Elsevier, 2009), Chap. 1, pp. 1-67.

24. N. Pavillon, C. S. Seelamantula, J. Kühn, M. Unser, and C. Depeursinge, "Suppression of the zero-order term in off-axis digital holography through nonlinear filtering," Appl. Opt. 48, H186-H195 (2009).

25. T. Kreis, Handbook of Holographic Interferometry: Optical and Digital Methods (Wiley-VCH, 2005).

26. L. Yu and M. K. Kim, "Wavelength-scanning digital interference holography for tomographic three-dimensional imaging by use of the angular spectrum method," Opt. Lett. 30, 2092-2094 (2005). 